Available online at http://jifnp.tp.ugm.ac.id
INDONESIAN FOOD AND NUTRITION PROCRESS

\title{
Penicillium Species Isolated From Cocoa, Coffee Beans, and Dried Cassava in Yogyakarta Indonesia and Their Ochratoxin Production
}

\author{
Mona Nur Moulia ${ }^{1)}$, Sigit Setyabudi ${ }^{1)}$, Baharuddin Salleh $^{2)}$, Endang S. Rahayu ${ }^{1^{*}}$ \\ 1) Faculty of Agricultural Technology, Universitas Gadjah Mada, Yogyakarta, Indonesia \\ ${ }^{2)}$ School of Biological Science, Universiti Sains Malaysia, Penang, Malaysia \\ ${ }^{*}$ Corresponding author, email address: endangsrahayu@ugm.ac.id
}

Received 15 May 2014; Accepted 5 August 2014; Published Online 10 September 2014

\begin{abstract}
The presence of Penicillium in cocoa and coffee beans, and dried cassava are detrimental due to its ability in ochratoxin A (OTA) production which carcinogenic and mutagenic to human. Objectives of this study were to isolate and identify Penicillium from cocoa, coffee beans and dried cassava in Yogyakarta by morphological and molecular characteristics, as well as to observe the ability of these isolates in OTA production on Yeast Extract Sucrose Agar (YES) medium. In this study, morphological characteristics were mainly based on the growth of isolates on identification media, while molecular characteristics were based on the similarity of PCR products using ITS4 and ITS5 as primers. OTA was detected by ELISA and UPLC methods. The result showed that 15 of 16 representative isolates obtained during this study were identified as Penicillium citrinum, one of the representative isolate from cocoa beans was identified as Penicillium paneum. Surprisingly, 13 among 15 of the obtained $P$. citrinum isolates from cocoa and coffee beans were positive in the production of OTA in YES medium, at the concentration of 4.64 to $25.26 \mu \mathrm{g} / \mathrm{g}$ media, while OTA was not detected in YES grown media by $P$. paneum and two isolates of $P$. citrinum from dried cassava. Conclusion of this study, the most found species Penicillium in cocoa and coffee beans were belong to $P$. citrinum which likely have a capability in the production of OTA.
\end{abstract}

Keywords: Penicillium, cocoa beans, coffee beans, dried cassava, OTA

\section{Introduction}

Cocoa and coffee beans, and cassava have an important role in Indonesia, especially in Yogyakarta, as a source of economic income. Mostly, drying and storage of cocoa, coffee beans and cassava are still using traditional method, such as drying the products out in the yard under the sun and storing in a humid warehouse condition. These suboptimal drying and storage processes render the products to be easily contaminated by filamentous fungi.

The presences of toxicogenic Penicillium species are generally responsible for the toxin content, even when fungal contamination were not observable anymore. Fungal contamination is suspected due to quite high moisture content $(>8 \%)$ and the slow sun drying process of the commodities. Several species of Penicillium are known to contaminate foods such as $P$. verrucosum on dried foods and cereals, $P$. nordicum on fermentation of meat and cheese which potential in OTA production (Larsen et al., 2001; Castella et al., 2002; Cabanes et al., 2010).

International Agency for Research on Cancer (IARC, 1982) mentioned that some Penicillium species capable of producing ochratoxin A (OTA) are dangerous because carcinogenic and mutagenic effects to humans and animals that consumed the contaminated food or feed. Recently the European Commission stated $2 \mathrm{~g}$ and $1 \mathrm{mg}$ for the maximum level of OTA in raw cacao and the processed products (Martinez-Culebras et al., 2008). 
Ochratoxin A (OTA) producing fungi are members of the genera Aspergillus and Penicillium. Species of Aspergillus, which produce OTA in cocoa are mainly Aspergillus carbonarius and $A$. niger (Mounjouenpou et al., 2008; Cabanes et al., 2010). Other important species regarding potential ochratoxin A production in coffee, rice, beverages and other foodstuffs are A. ochraceus, A. westerdijkiae, and A. steynii (Frisvad et al., 2004). While from genus Penicillium, at the moment only two species accepted as OTA producing, i.e., $P$. verrucosum and $P$. nordicum (Larsen et al., 2001; Castella et al., 2002; Cabanes et al., 2010). While according to Vega et al., (2006) among 11 Penicillium species endophytic in coffee plants, four isolates produced OTA A, i.e., $P$. brevicompactum, $P$. crustosum, $P$. olsonii and $P$. oxalicum.

Study on OTA contamination during fermentation and drying of cocoa beans at Ivory Coast, Dano et al., (2013) reported that OTA was detected in beans at all stages of post-harvesting operations, such as pod-opening, fermentation, drying, and storage. Our previous paper showed that black Aspergillus was the dominant species of Aspergillus in cocoa and coffee beans, and we found that $A$. carbonarius was the OTA producer among the black Aspergillus (Nugroho et al., 2013). Our concern in this study was to characterize the species of Penicillium that also contaminate cacao and coffee beans, and dried cassava in Yogyakarta and to determine their potency in the production of OTA.

\section{Materials and Methods Isolation of Penicillium}

Filamentous fungi Penicillium were isolated from cocoa and coffee beans, and cassava that have undergone drying process. Samples were collected from several farmers, $1-3 \mathrm{~kg}$ of samples were collected each of them. Cocoa and coffee beans samples were collected from Kulonprogo, while the dried cassava samples from Gunung Kidul, Yogyakarta. All of the samples were plated by direct and dilution methods as described by Samson et al., (2004). Media for plating were Dichloran Rose Bengal Chloramphenicol Agar (DRBC, Oxoid) and Dichloran Gliserol-18 Agar (DG18 , Oxoid). The plates were incubated for 7 days in room temperature. Pure culture of Penicillium were transferred to Malt Extract Agar (MEA) slant tubes, incubated for 7 days, and were stored at 4 ${ }^{\circ} \mathrm{C}$ for further study.

\section{Identification of Fungi}

Primary identification of Penicillium isolates was done based on macroscopic and microscopic characteristics (Samson et al., 2004). Single spore of the isolates were inoculated at three points of identification media, and incubated for 7 days at $25^{\circ} \mathrm{C}$. Identification used media were Malt Extract Agar (MEA), Czapek Yeast Extract Agar (CYA), and Yeast Extract Sucrose agar (YES). Isolates were then grouped according to their similarity of macroscopic and microscopic characteristics, and one was selected from each group as representative isolates for further study.

\section{Extraction of DNA and PCR Amplification}

Molecular analysis for species identification of Penicillium isolates was based on the similarity of amplicon using ITS4 and ITS5 as primers. Isolates were grown in Potato Dextrose (PD) broth for 7 days to obtain genomic DNA. The mycelia were harvested and grinded into fine powder after freezing with liquid nitrogen. DNA was extracted with DNeasy Plant Qiagen, Valencia (followed manufacturer's directions). DNA was then amplified by PCR using ITS4 and ITS5 as primers (Bisbal et al., 2009). PCR amplification was performed with a volume of $25 \mu \mathrm{L}$ consisting of GoTaq $^{\circledR} 5 x$ buffer (Promega), GoTaq ${ }^{\circledR}$ DNA polymerase $(5 \mu / \mu \mathrm{L})$ (Promega), $25 \mathrm{mM} \mathrm{MgCl} 2$, dNTP (Promega) for each primer and DNA isolates.

PCR analysis was performed using a thermal cycler (Peltier PTC-100) with the parameters of 1 cycle of pre-denaturation $5 \mathrm{~min}$ at $95^{\circ} \mathrm{C} ; 30$ cycles consisting of $30 \mathrm{~s}$ at $95^{\circ} \mathrm{C}$ for denaturation, $30 \mathrm{~s}$ at $58^{\circ} \mathrm{C}$ for annealing, and $1 \mathrm{~min}$ at $72^{\circ} \mathrm{C}$ for extension; followed by 1 cycle of final extension 5 min at $72^{\circ} \mathrm{C}$ (Bisbal et al., 2009). PCR products were separated by electrophoresis using $1 \%$ agarose; containing Ethidium Bromide (EtBr) for staining. DNA bands were visualized under ultraviolet (UV) light, and sizes were estimated by comparison with a DNA standard length (TM GeneRuler 1kbp DNA ladder, MBI Fermentas, Lithuania).

\section{Sequencing Analysis}

PCR products were purified using the QIAquick PCR Purification Kit, Qiagen, Valencia (followed the manufacturer's instruction). Nitrogenous bases sequencing of purified products were performed in the Center of Chemical Biology, Universiti Sains of Malaysia (USM). All of the DNA sequence of the isolates 
were matched for similarity using BLAST to NCBI. All the isolates were also analyzed for network joining (NJ) using bootstrap method and the maximum composite likelihood with Mega version 4.0 software. Penicillium maleagrinum Angg-PBB613 was used as an out-group.

\section{Production of OTA}

Isolates were grown in YES medium and incubated for at $25^{\circ} \mathrm{C}$ for 7 days to determine the ability of Penicillium isolates in producing OTA (Accensi et al., 2004). Three agar plugs were removed from different points of the colony (about $5 \mathrm{~g}$ ) from each culture. Samples were analyzed for OTA using RIDASCREEN ${ }^{\circledR}$ OTA ELISA test kits ( $r$-BioPharm). All sample preparation and test procedures followed the manufacturer's instructions. Limit of detection is about $2.5 \mathrm{ppb}$. For double check, OTA was also detected by UPLC. Three agar plugs of YES medium grown by isolates were removed and extracted by $0.5 \mathrm{~mL}$ of methanol. After centrifugation, the supernatant was analyzed by utilizing UPLC.

\section{Results and Discussion Isolation of Penicillium}

This study was succeeded in obtaining 67 isolates of the filamentous fungi of Penicillium in cacao and coffee beans, and cassava samples. The isolates were grouped according to their colony appearance in MEA, CYA and YES, and from each group isolates were taken as their representative for further study.

Fungal contamination in cacao and coffee beans, and cassava were further characterized by $a_{w}$ value of samples at a range between 0.81 and 0.94 . The $a_{w}$ values were optimum for the growth of species of the genera Aspergillus and Penicillium which have minimum $\mathrm{a}_{\mathrm{w}}$ requirements of 0.75 to 0.86 (Pitt and Hocking, 2009; Esteban et al., 2006).

\section{Macroscopic and Microscopic Characteristics}

Sixteenth selected Penicillium isolates were identified by macroscopic and microscopic characteristics (Samson, et al., 2004; Pitt and Hocking, 2009). The grown colony diameter of fifteen isolates (Y-PK-6, Y-PG-4, Y-PK-9, Y-PK-10, YPK-29, Y-PK-16, Y-PG-9, Y-PK-17, Y-PC-5, Y-PK-20, Y-PK-14, Y-PK-31, Y-PK-7, Y-PC-3, Y-PK-24) that on MEA medium were in the range of $22-30 \mathrm{~mm}$. Mycelia were white or yellow orange, clear- colored exudates, velvety and floccose. Colonies on CYA medium were larger, up to $30 \mathrm{~mm}$, white mycelia in the middle, sometimes yellow, and produced yellow soluble pigment, has smoothwalled conidiophores with stipa 100-300 $\mu \mathrm{m}$ long, globose to subglobose-shaped conidia with a diameter of $2.2-3.0 \mu \mathrm{m}$, flask-shaped phialide and biverticillate conidiophores. These isolates were suspected to be Penicillium citrinum.

Isolate Y-PC-12 has a very rapid growth on MEA medium (37-47 mm diameter), dull-green colored colonies, and pale reverse. In CYA medium the colony diameter was $28-29 \mathrm{~mm}$, pale green to dark blue green with velvety surface, biverticillate conidiophores, rough stipa, rough-walled phialide, smooth-walled globose shaped conidia. Isolate $\mathrm{Y}$ PC-12 was suspected as Penicillium paneum. Odserve and reverse appearance of Penicillium colonies and Penicillium maleagrinum AngPBB6-13 was used as control (negative OTA producers) in this study as presented in Fig. 1.

\section{Molecular Identification}

DNA were amplified using PCR using ITS4 and ITS5 as pair primer as reported in GonzalesSalgado et al., (2005); Patino et al., (2005); and Bisbal et al., (2009). The PCR result of the amplified ITS region in this study was about 560 base pairs (Fig. 2). The result of this amplification was similar to the results by Bisbal et al., (2009), Gonzales-Salgado et al., (2005), Patino et al., (2005), and Duponta et al., (1999), which were 650 bp, 612 bp, 603 bp and 498 bp, respectively.

Variation in size of the amplified fragment was caused by the rDNA length of the ITS regions. Variation of ITS sequences in this region allows the use of this region for phylogenetic study of many different organisms (Ahmed et al., 1999). Some very significant variations can occur because of deletions, insertions, or substitutions between species in the ITS (Peay et al., 2008). The similarity for species identification was done by comparing the sequence of nucleotide bases in the ITS region of isolates with ITS region of Penicillium species that exist in the GenBank, then the sequence of nucleotide bases in http://blast.ncbi.nlm.nih.gov/Blast were read using BLAST. BLAST results and comparison of isolates of Penicillium nitrogenous base sequences in the ITS region (\% of similarity) can be seen in Table 1. BLAST results show that all 


\begin{tabular}{|l|l|l|l|}
\hline \multicolumn{1}{|c|}{ Isolate } & MEA & CYA & YES \\
\hline Y-PK-10 & & & \\
\hline
\end{tabular}

Fig. 1 Odserve and reverse appearance of Penicillium colonies

the genes have similarity with other fungal species ITS region. This indicates that the PCR was successfully amplified ITS region. Sampietro et al., 
(2010) stated that molecular analysis could identify Penicillium and Fusarium species when the base pair sequence had high homology (98$100 \%$ of amino acids).

To support the results of morphology and BLAST analysis, the species similarity analysis can be done by analyzing the sequence of nitrogenous bases with a multi-alignment of ITS regions. The results of sequence alignment Penicillium that based on the nucleotide bases Multalin can be seen in Fig. 4. Base sequence of isolate Y-PK-6, Y-PK-9, Y-PK-14, Y-PG-4, Y-PK-10, Y-PK-24, Y-PK-20, Y-PK-16, Y-PG-9, Y-PK-17, Y-PK31, Y-PK-7, Y-PC-3, Y-PC-5 were different from YPC-12 and Ang-PBB6-13 in sequence number 64, 121-123, and 222-260 (Fig. 3). Fadiloglu and Erkmen (1999) suggested the possibility that differences in amino acid affect the shape, structure, and reactivity of these proteins.

The alignment performed with the aim to determine the degree of homology of DNA base sequences analysis, to find out the differences and similarities as well as the sequence of nucleotide base composition (Whitford, 2005). The alignment results showed a high degree of homology between the samples studied. Due to the nature of ITS4 and ITS5 variation; this juxtaposition appears in the gap (indicated by dashed lines). The existence of this gap indicates a high occurrence of mutations either in the form of insertions and deletions (Hidayat et al., 2008). Nevertheless the results of Clustal-W alignment previously demonstrated a high degree of homology (96-99\%) between genes in the cluster.

The resulting phylogenetic tree by neighbor joining method bootstrap consensus suggests kinship between each species (Kristensen et al., 2005) with bootstrap support values are quite large, amounting to 96 can be seen in Fig. 4 . Phylogenetic indicated that 15 isolates (Y-PK-6, YPG-4, Y-PK-9, Y-PK-10, Y-PK-29, Y-PK-16, Y-PG-9, Y -PK-17, Y-PC-5, Y-PK-20, Y-PK-14, Y-PK-31, Y-PK-7, Y-PC-3, Y-PK-24) were identified as Penicillium citrinum, 1 isolate (Y-PC-12) as Penicillium paneum, and 1 isolates (Ang-PBB6-13) as Penicillium maleagrinum.

Phylogenetic analysis was used to identify species and show the relation among species of Penicillium. Identification of the isolates demonstrated through the same branch with reference strains from GenBank database. Phylogenetic tree in Fig. 4 shows compliance with the previous molecular identification. The tree may also indicate differences among Penicillium citrinum, Penicillium paneum and Penicillium maleagrinum based on different branches.

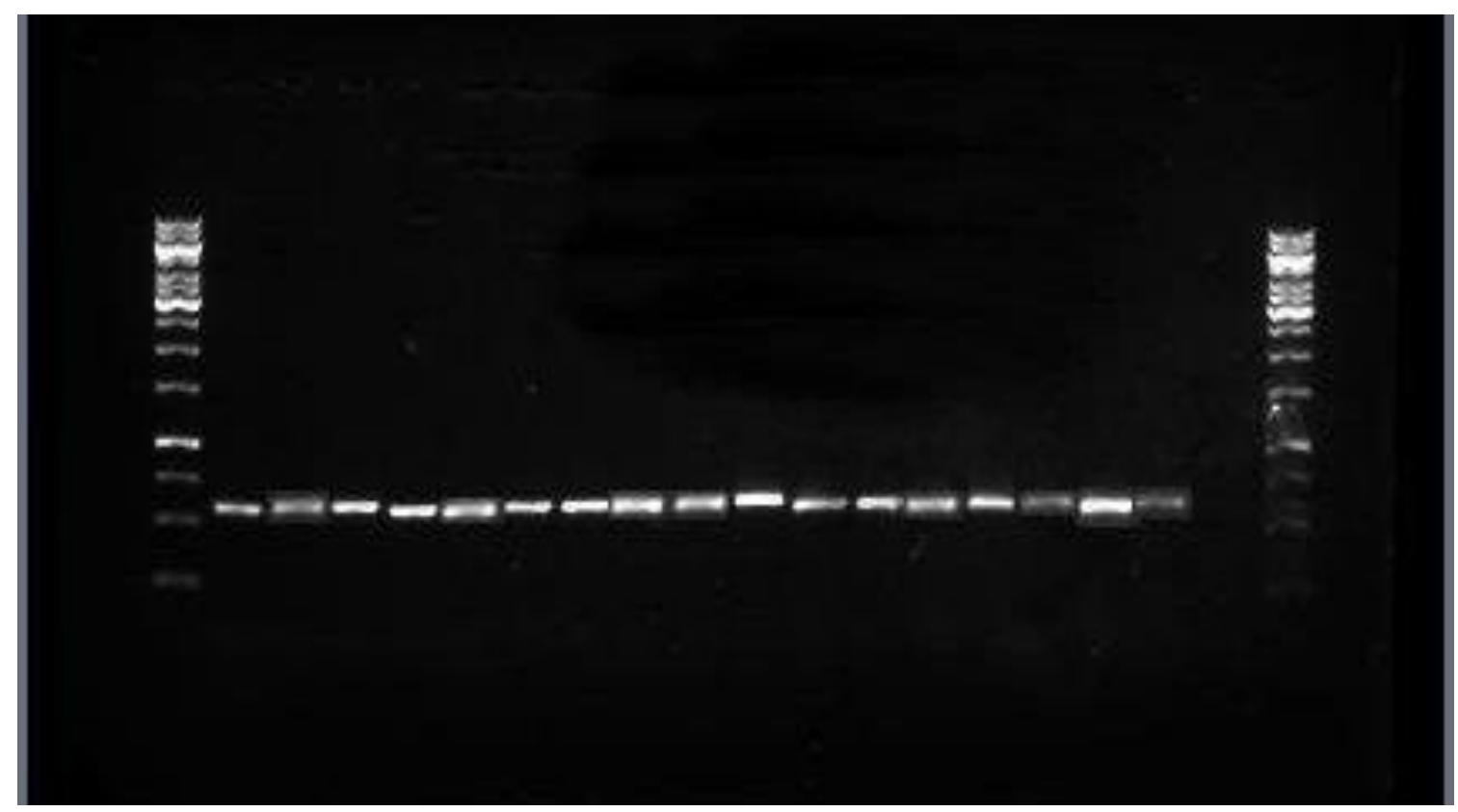

Fig. 2 The amplification of Penicillium isolates using Primer ITS4 and ITS5 the above isolates 1-17, C = control Description: 1) Y-PK-31, 2) Y-PK-29, 3) Y-PC-12, 4) Y-PG-9, 5) Y-PK-17, 6) Y-PG-4, 7) Y-PK-14, 8) Y-PK-6, 9) Y-PK-10, 10) Ang PBB6-13, 11) Y-PK-24, 12) Y-PK 20, 13) Y-PK-7, 14) Y-PC-5, 15) Y-PK-16, 16) Y-PC-3, 17) Y-PK-9 
Table 1. BLAST results and comparison of isolates of Penicillium nitrogen base sequences in the ITS region (\% of similarity)

\begin{tabular}{|c|c|c|c|c|}
\hline No & Isolate & $\begin{array}{l}\text { ITS Sequences generated by PCR compared to } \\
\text { ITS sequences published in the Genbank }\end{array}$ & Similarity & $\begin{array}{c}\text { Accession } \\
\text { Number }\end{array}$ \\
\hline \multirow[t]{2}{*}{1.} & Y-PC-3 & Penicillium citrinum strain DTO & $100 \%$ & GU944575 \\
\hline & & Penicillium citrinum strain UASWS0339 & $100 \%$ & HM235979 \\
\hline \multirow[t]{2}{*}{2.} & Y-PC-5 & Penicillium citrinum strain S36 & $100 \%$ & JF266706 \\
\hline & & Penicillium griseofulvum strain F26 & $100 \%$ & EU497956 \\
\hline \multirow[t]{2}{*}{3.} & Y-PC-12 & Penicillium paneum strain CBS 112296 & $99 \%$ & HQ442339 \\
\hline & & Penicillium paneum strain CBS 101032 & $99 \%$ & HQ442346 \\
\hline \multirow[t]{3}{*}{4.} & Y-PK-6 & Penicillium citrinum strain NRRL 35449 & $100 \%$ & DQ123646 \\
\hline & & Penicillium griseofulvum strain L12 & $100 \%$ & EU780695 \\
\hline & & Penicillium westlingii strain NRRL 800 & $100 \%$ & AF033423 \\
\hline \multirow[t]{2}{*}{5.} & Y-PK-7 & Penicillium citrinum strain KUC3084 & $98 \%$ & HM469428 \\
\hline & & Penicillium griseofulvum strain 091402 & $98 \%$ & EU664471 \\
\hline \multirow[t]{2}{*}{6.} & Y-PK-9 & Penicillium citrinum strain S36 & $100 \%$ & JF266706 \\
\hline & & Penicillium griseofulvum strain F26 & $100 \%$ & EU497956 \\
\hline \multirow[t]{2}{*}{7.} & Y-PK-10 & Penicillium citrinum strain UASWS0352 & $100 \%$ & HM235992 \\
\hline & & Penicillium griseofulvum strain L12 & $100 \%$ & EU780695 \\
\hline \multirow[t]{3}{*}{8.} & Y-PK-14 & Penicillium citrinum strain S36 & $100 \%$ & JF266706 \\
\hline & & Penicillium griseofulvum strain F26 & $100 \%$ & EU497956 \\
\hline & & Penicillium westlingii strain NRRL 800 & $100 \%$ & AF033423 \\
\hline \multirow[t]{2}{*}{9.} & Y-PK-16 & Penicillium citrinum strain T134 & $99 \%$ & JF793520 \\
\hline & & Penicillium griseofulvum strain L12 & $99 \%$ & EU780695 \\
\hline \multirow[t]{2}{*}{10.} & Y-PK-17 & Penicillium citrinum strain OY30207 & $98 \%$ & FJ571468 \\
\hline & & Penicillium griseofulvum strain L12 & $98 \%$ & EU780595 \\
\hline \multirow[t]{2}{*}{11.} & Y-PK-20 & Penicillium citrinum strain JO9 Penicillium & $98 \%$ & EU645709 \\
\hline & & griseofulvum strain KUC5017 & $98 \%$ & GQ241285 \\
\hline \multirow[t]{2}{*}{12.} & Y-PK-24 & Penicillium citrinum strain DTO 58A4 & $99 \%$ & GU944575 \\
\hline & & Penicillium griseofulvum strain L3 & $99 \%$ & EU780694 \\
\hline \multirow[t]{2}{*}{13.} & Y-PK-29 & Penicillium citrinum strain OY30207 & $99 \%$ & FJ571468 \\
\hline & & Penicillium griseofulvum isolate NW440 & $99 \%$ & EU520067 \\
\hline \multirow[t]{2}{*}{14.} & Y-PK-31 & Penicillium citrinum strain CBS 122396 & $99 \%$ & GU944574 \\
\hline & & Penicillium westlingii strain NRRL 800 & $99 \%$ & AF033423 \\
\hline \multirow[t]{2}{*}{15.} & Y-PG-4 & Penicillium citrinum strain S36 & $99 \%$ & JF266706 \\
\hline & & Penicillium griseofulvum strain F26 & $99 \%$ & EU497956 \\
\hline \multirow[t]{2}{*}{16.} & Y-PG-9 & Penicillium citrinum strain KACC43900 & $98 \%$ & EU821333 \\
\hline & & Penicillium griseofulvum strain L12 & $98 \%$ & EU780695 \\
\hline 17. & Ang PBB6-13 & $\begin{array}{l}\text { Penicillium meleagrinum var. viridiflavum } \\
\text { strain KUC1678 }\end{array}$ & $99 \%$ & HM469412 \\
\hline
\end{tabular}

\section{Ochratoxin A Producing Ability}

All 16 selected isolates were tested for their ability to produce OTA. The results are shown in Table 2. Ochratoxin analysis using ELISA ( $L O D=2.5 \mu \mathrm{g} / \mathrm{g}$ ) from YES agar medium show that 13 isolates of isolated Penicillium from cocoa and coffee beans were able to produce OTA from 4.65 to $25.26 \mu \mathrm{g} / \mathrm{g}$ media. These isolates were supposed to be $P$. citrinum. Two isolates also supposed to be $P$. citrinum (Y-PG-4 and Y-PG-9) from dried cassava, while one isolates Y-PC-12 that supposed to be $P$. paneum from cocoa beans have no capability in producing OTA. $P$. paneum known to be non OTA producer (Mounjouenpou et al., 2008), however, there is no publication at the moment that mentioned $P$. citrinum has a capability in producing OTA, only two species of Penicillium, i.e., $P$. verrucosum and $P$. nordicum in this study, detection of OTA was double checked with UPLC, and the result was similar to ELISA methods.

The used method for determination of OTA was ELISA. ELISA is a reliable and rapid method for OTA determination in various kinds of commodities (Fujii et al., 2007; Matrella et al., 2006; Zheng et al., 2005). ELISA was preferred because it requires less sample volume and sample preparation is faster than other conventional methods such as TLC and HPLC (Zheng et al., 2005). This method is very sensitive to mycotoxins that was extracted from the natural matrix (White and Johnson, 2003). 


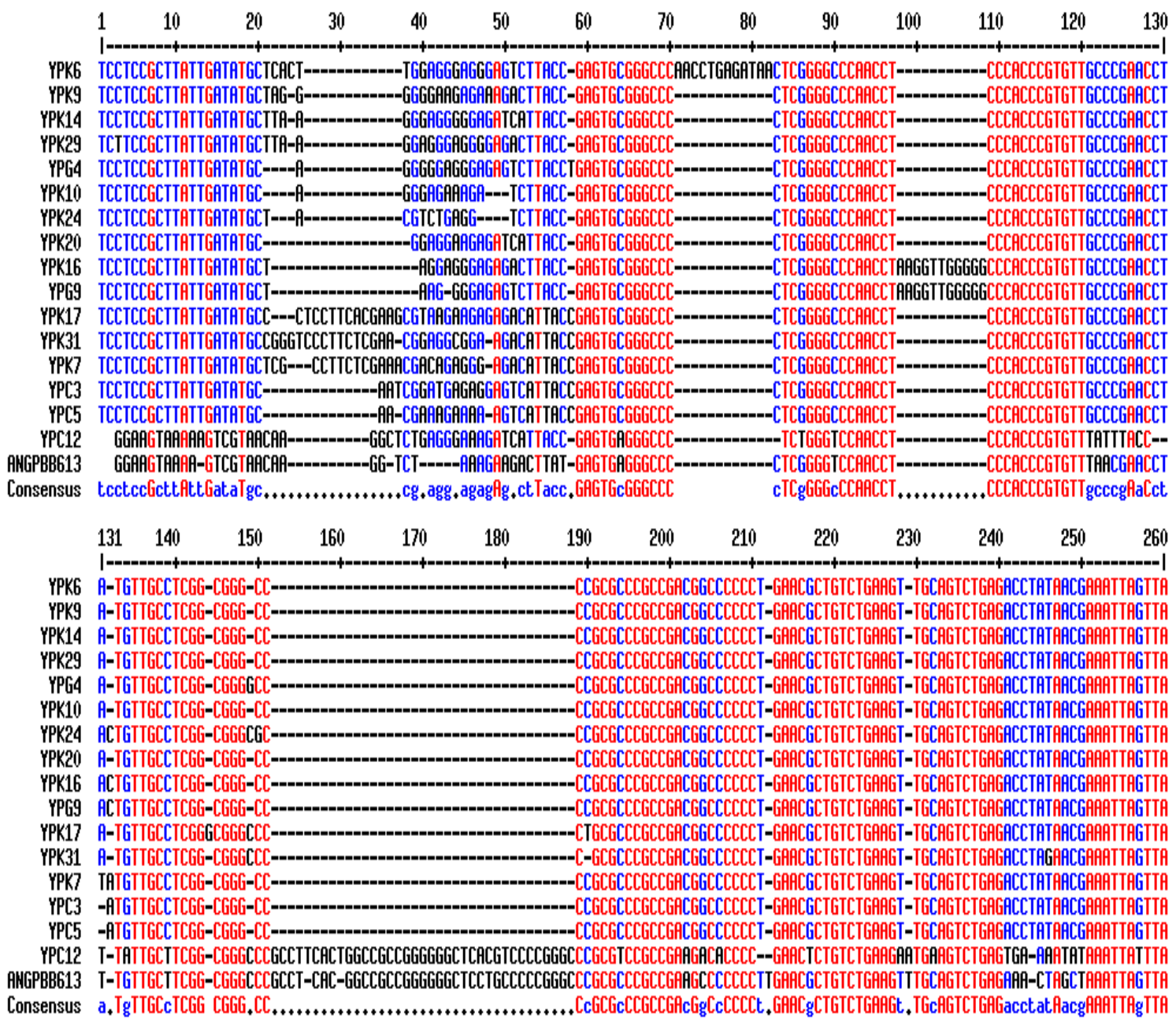

Fig. 3. Alignment of nucleotide base sequence of Penicillium based Multalin

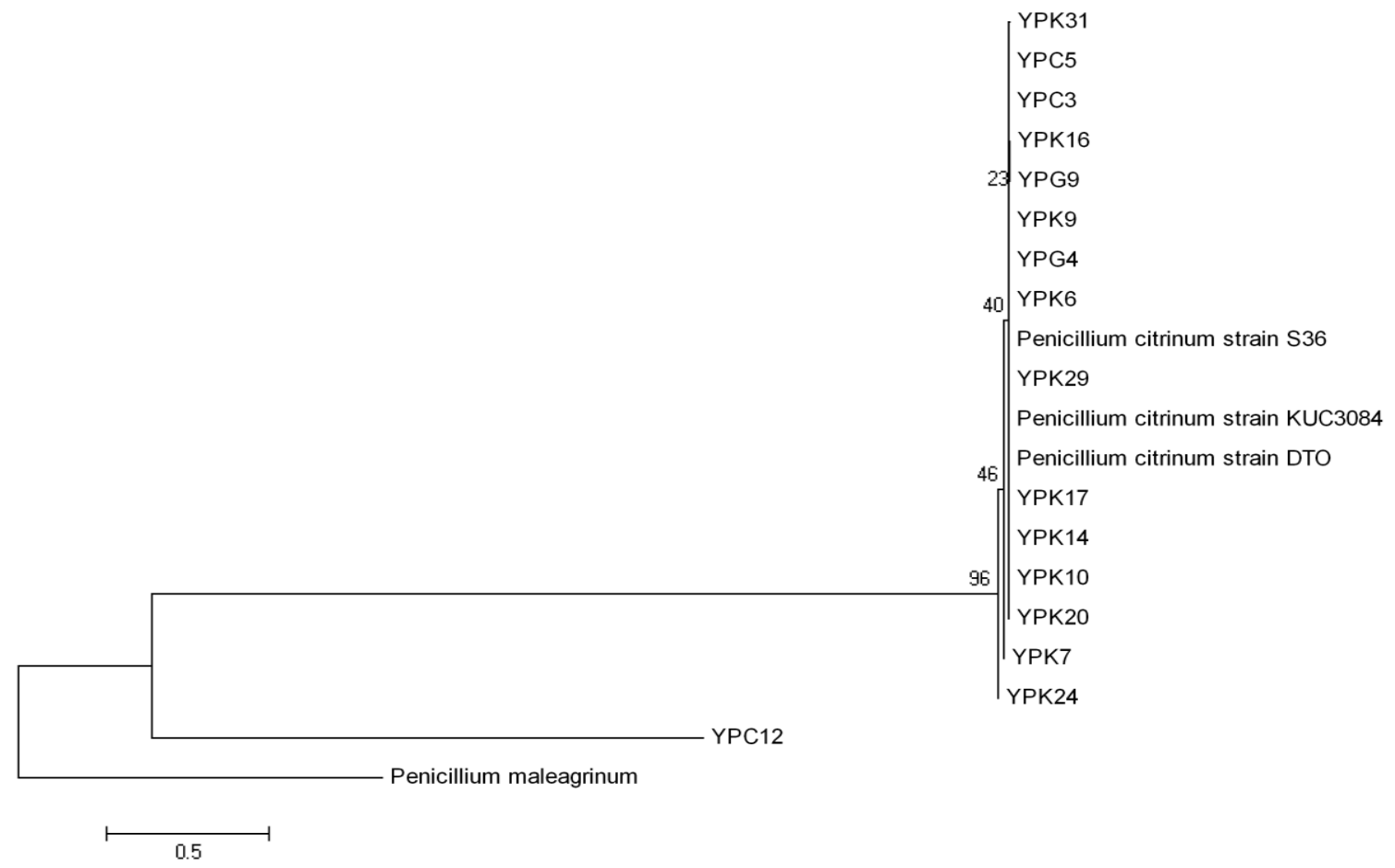

Fig. 4 Penicillium species phylogenetic tree 
Table 2. Ochratoxin A content on YES medium in order of 16 isolates were selected

\begin{tabular}{llcc}
\hline No & Isolate & Source of isolate & OTA Concentration $(\mu \mathrm{g} / \mathrm{g})$ \\
\hline 1. & Y-PC-3 & Cocoa beans & 25,26 \\
2. & Y-PC-5 & Cocoa beans & 4,65 \\
3. & Y-PC-12 & Cocoa beans & $<$ LOD \\
4. & Y-PK-6 & Coffee beans & 4,72 \\
5. & Y-PK-7 & Coffee beans & 14,96 \\
6. & Y-PK-9 & Coffee beans & 25,09 \\
7. & Y-PK-10 & Coffee beans & 5,26 \\
8. & Y-PK-14 & Coffee beans & 11,14 \\
9 & Y-PK-16 & Coffee beans & 5,26 \\
10. & Y-PK-17 & Coffee beans & 21,12 \\
11. & Y-PK-20 & Coffee beans & 6,30 \\
12. & Y-PK-24 & Coffee beans & 5,71 \\
13. & Y-PK-29 & Coffee beans & 19,17 \\
14. & Y-PK-31 & Coffee beans & 13,73 \\
15. & Y-PG-4 & Dried cassava & $<$ LOD \\
16. & Y-PG-9 & Dried cassava & $<$ LOD \\
17. & Ang PBB6-13 & as control & $<$ LOD \\
\hline
\end{tabular}

However, it should be noted that ELISA method uses enzymes, which is quite complex because its activity is influenced by various factors that can interfere with the binding of toxins and mycotoxins in samples, causing overor underestimation (Asihara and Kasahara, 2001). Visconti et al., (1999) mentioned that problems of OTA analysis in red wine may be due to the presence of several anthocyanin and other pigments that interfere with OTA-binding to the antibody so as to provide false positive results. In this study the above problems were not found as components of YES media avoid the potential of crosslinking.

\section{Conclusion}

Penicillium citrinum was the dominant contaminant in cacao and coffee beans, and cassava. Macroscopic, microscopic, and molecular level data were required to be able to accurately identify the species of Penicillium. Nitrogen base sequences of ITS region proved specific and sensitive enough to distinguish species of Penicillium.

In this study 13 among 15 of $P$. citrinum isolates obtained from cocoa and coffee beans were positive in the production of OTA in YES medium, at the concentration of 4.64 to 25.26 $\mu \mathrm{g} / \mathrm{g}$ media, while OTA was not detected in YES grown media by $P$. paneum and two isolates of $P$. citrinum from dried cassava.

\section{References}

Accensi, F., Abarca, M.L., and Cabanes, F.J. 2004. Occurrence of Aspergillus species in mixed feeds and component raw materials and their ability to produce ochratoxin A. Food Microbiol. 21: 623-627.

Ahmed, A.O.A., Mukhtar, M.M., Kools-Sijmons, M., Fahal, A.H., De Hoog, S., Ende van den B.G., Zijlstra, E.E., Verbrugh, H., Abugrou, E.S., and Elhassan, A.M. van A. Belkum. 1999. Development of a species-specific PCRrestriction fragment length polymorphism analysis procedure for identification of Madurella mycetomatis. J. Clin. Microbiol. 37: 3175-3178.

Asihara, Y. and Kasahara, Y. 2001. Immunoassay and Immunochemistry. In John, B.H (eds), Clinical diagnosis and management by laboratory methods $21^{\text {st }}$ ed. Philadelphia.

Bisbal, F., Vicente, J.G., Ramon, D., and MartinezCulebras, P.V. 2009. ITS_RFLP characterization of black Aspergillus isolates responsible for ochratoxin A contamination in cacao beans. J. Eur. Food Res. Technol. 229: 751-755.

Cabanes, F.J., Bragulat, M.R., and Castellá, G. 2010. Ochratoxin A producing species in the genus penicillium. Toxins. 2:1111-1120.

Castellá, G., Larsen, T.O., Cabanes, J., Schmidt, H., Alboresi, A., Niessen, L., Farber, P., and Geisen, R., 2002. Molecular characterization of ochratoxin A producing strains of the genus Penicillium. Syst. Appl. Microbiol. 25: 74-83. 
Dano, S.D., Manda, P., Dembele, A., Abla, A.M.K., Bibaud, J.H., Gouet, J.Z., and Sika, C.B.Z. 2013. Influence of fermentation and drying materials on the contamination of cocoa beans by ochratoxin A. Toxins. 5: 2310-2323.

Duponta, J., Magnina, S., Martib, A., and Brousse, M. 1999. Molecular tools for identification of Penicillium starter cultures used in the food industry. Int. J. Food Microbiol. 49: 109-118.

Esteban, A., Abarca, M.L., Bragulat, M.R., and Cabanes, F.J. 2006. Effect of water activity on ochratoxin A production by Aspergillus niger aggregate species. Int. J. Food Microbiol. 108 : 188-195.

Fadıloglu, S. and Erkmen, O. 1999. Lipase production by Rhizopus oryzae growing on different carbon and nitrogen sources. J. Sci. Food Agric. 79: 1936-1938.

Frisvad, J.C., Frank, J.M., Houbraken, J.A.M.P., Kuijpers, A.F.A., and Samson, R.A. 2004. New Ochratoxin A producing species of Aspergillus section circumdati. Stud. Mycol. 50: 23-43.

Fujii, S., Ono, E.Y.S., Ribeiro, R.M.R., Assuncao, F.G.A., Takabayashi, C.R., Oliveira, T.C.R.M., Itano, E.N., Ueno, Y., Kawamura, O., and Hirooka, E.Y. 2007. A comparison between enzyme immunoassay and HPLC for ochratoxin A detection in green, roasted and instant coffee. Braz. Arch. Biol. Techn. 50: 349-359.

Gonzalez-Salgado, A., Patino, B., Vasquez C., and Gonzalez-Jaen, M.T. 2005. Discrimination of Aspergillus niger and other Aspergillus species belonging to section Nigri by PCR assays. FEMS Microbiol. Lett. 245: 353-361.

Hamelin, R.C., Berube, P., Gignac, M., and Bourassa, M. 1996. Identification of root fungi in nursery seedlings by nested multiplex PCR. Appl. Environ. Microb. 11: 4026-4031.

Henry, T., Iwen, P.C., and Hinrichs, S.H. 2000. Identification of Aspergillus species using internal transcribed spacer regions 1 and 2 . J. Clin. Microbiol. 38: 1510-1515.

Hidayat, T., Kusumawaty, D., Kusdianti, Yati, D.D., Muchtar, A.A., and Mariana, D. 2008. Using the base sequence of DNA Internal Transcribed Spacer Region (ITS). Education Department of Biology, Faculty of Mathematics and Science Education University of Indonesia, Bandung.

IARC. 1982. Ochratoxin A. Monographs on the evaluation of carcinogenic risk to humans. Lyon, France, IARC Working Group. World Health Organization, pp. 250-277.
Kristensen, R., Torp, M., Kosiak, B., and HolstJensen, A. 2005. Phylogeny and toxigenic potential is correlated in Fusarium species as revealed by partial translation elongation factor 1 alpha gene sequences. Mycol. Res. 109 (2), 173-186.

Larsen, T.O., Svendsen, A., and Smedsgaard, J. 2001. Biochemical characterization of ochratoxin A-producing strains of the genus Penicillium. App. Environ. Microb. 67: 36303635.

Martinez-Culebras, P.V., Ramon, D., Bisbal, F., Gil, J.V., and Sanchez-Hervas, M. 2008. Mycobiota and mycotoxin producing fungi from cacao beans. Int. J. Food Microbiol. 125: 336-340.

Matrella, R., Monaci, L., Milillo, M.A., Palmisano, F., and Tantillo, M.G. 2006. Ochratoxin A determination in paired kidneys and muscle samples from swines slaughtered in southern Italy. Food Control. 17: 114-117.

McCulloug, M.J., Clemons, K.V., McCusker, J.H., and Stevens, D.A. 1998. Intergenic transcribed spacer PCR ribotyping for differentiation of Saccharomyces species and interspecific hybrids. J. Clin. Microbiol. 36: 1035-1038.

Mounjouenpou, P., Gueule, D., Fontana-Tachon, A., Guyot, B., Tondje, P.R., and Guiraud, J.P. 2008. Filamentous fungi producing ochratoxin a during cocoa processing in Cameroon. Int. J. Food Microbiol. 121: 234-241.

Nugroho, A.D., Setyabudi, F.M.C.S., Salleh, B., and Rahayu, E.S. 2013. Ochratoxigenic black aspergilli isolated from dried agricultural products in Yogyakarta, Indonesia. J. Food Sci. Eng. 3:472-480.

Sampietro, D.A., Marin, P., Iglesias, J., Presello, D.A., Vattuone, M.A., Catalan, C.A.N., and Jaen, M.T.G. 2010. A Molecular based strategy for rapid diagnosis of toxigenic fusarium species associated to cereal grains from Argentina. Fungal Biol. 114: 74-81.

Samson, R.A., Hoekstra, E.S., and Frisvad, J.C. 2004. Introduction to Food and Airborne Fungi, 7th ed. Central bureauvoor Schimmelcultures, Utrecht, Netherlands.

Sánchez-Hervás, M., Gil, J.V., Bisbal, F., Ramón, D., and Martínez-Culebras, P.V. 2008. Mycobiota and mycotoxin producing fungi from cocoa beans. Int. J. Food Microbiol. 125:336-340.

Patino, B., Gonzalez-Salgado, A., Vasquez, C., and Gonzalez-Jaen, M.T. 2005. PCR detection assays for the ochratoxin $A$ producing 
Aspergillus carbonarius and Aspergillus ochraceus species. Int. J. Food Microbiol. 104:207-214.

Peay, K.G., Kennedy, P.G., and Bruns, T.D. 2008. Fungal community ecology: a hybrid beast with a molecular master. BioScience. 58: 799810.

Pitt, J.I. and Hocking, A.D. 2009. Fungi and Food Spoilage. Springer. New York. USA.

Vega, F.E., Posada, F., Peterson, S.W., Gianfagna, T.J., and Chaves, F. 2006. Penicillium species endophytic in coffee plants and ochratoxin $A$ production. Mycologia. 98 (1): 31-42.

Visconti, A., Pascale, M., and Centonze, G. 1999. Determination of ochratoxin $A$ in wine by means of immunoaffinity column clean-up and high-performance liquid chromatography. J. Chromatogr. A. 864: 89-101.

White, P.J. and Johnson, L.A. 2003. Corn: Chemistry and Technology. American Association of Cereal Chemistry, Inc. USA.

Whitford, D. 2005. Protein : Structure and Function. John Wiley and Sons Ltd. England.

Zheng, Z., Hanneken, J., Houchins, D., King, R.S., Lee, P., and Richard, J.L. 2005. Validation of an ELISA test kit for the detection of ochratoxin $A$ in several food commodities by comparison with HPLC. Mycopathologia. 159: 265-272. 